\title{
The Role of Timing in the Induction of Neuromodulation in Perceptual Learning by Transcranial Electric Stimulation
}

\author{
Cornelia Pirulli ${ }^{a}$, Anna Fertonani ${ }^{a}$, Carlo Miniussi ${ }^{a, b, *}$ \\ ${ }^{\text {a }}$ Cognitive Neuroscience Section, IRCCS Centro San Giovanni di Dio Fatebenefratelli, Brescia, Italy \\ ${ }^{\mathrm{b}}$ Department of Clinical and Experimental Sciences, National Institute of Neuroscience, University of Brescia, Brescia, Italy
}

\section{A R T I C L E I N F O}

\section{Article history:}

Received 20 September 2012

Received in revised form

21 December 2012

Accepted 21 December 2012

Available online 28 January 2013

\section{Keywords:}

tES

Transcranial direct current stimulation

(tDCS)

Transcranial random noise stimulation (tRNS)

Neuroplasticity

Visual perceptual learning (VPL)

Cognition

Non-invasive brain stimulation (NIBS)

\begin{abstract}
A B S T R A C T
Background: Transcranial electric stimulation (tES) protocols are able to induce neuromodulation, offering important insights to focus and constrain theories of the relationship between brain and behavior. Previous studies have shown that different types of tES (i.e., direct current stimulation - tDCS, and random noise stimulation - tRNS) induce different facilitatory behavioral effects. However to date is not clear which is the optimal timing to apply tES in relation to the induction of robust facilitatory effects. Objective/hypothesis: The goal of this work was to investigate how different types of tES (tDCS and tRNS) can modulate behavioral performance in the healthy adult brain in relation to their timing of application. We applied tES protocols before (offline) or during (online) the execution of a visual perceptual learning (PL) task. PL is a form of implicit memory that is characterized by an improvement in sensory discrimination after repeated exposure to a particular type of stimulus and is considered a manifestation of neural plasticity. Our aim was to understand if the timing of tES is critical for the induction of differential neuromodulatory effects in the primary visual cortex (V1).

Methods: We applied high-frequency tRNS, anodal tDCS and sham tDCS on V1 before or during the execution of an orientation discrimination task. The experimental design was between subjects and performance was measured in terms of $d^{\prime}$ values.

Results: The ideal timing of application varied depending on the stimulation type. tRNS facilitated task performance only when it was applied during task execution, whereas anodal tDCS induced a larger facilitation if it was applied before task execution.

Conclusion: The main result of this study is the finding that the timing of identical tES protocols yields opposite effects on performance. These results provide important guidelines for designing neuromodulation induction protocols and highlight the different optimal timing of the two excitatory techniques.
\end{abstract}

(c) 2013 Elsevier Inc. All rights reserved.

\section{Introduction}

Transcranial electric stimulation (tES) is a method that has attracted significant attention because it can modulate human brain activity $[1,2]$. Indeed the application of tES is thought to induce neuromodulation, as shown by improvements in behavioral and cognitive performance in normal and pathological subjects [3]. Behavioral induced neuromodulation has been observed during perceptual learning (PL) in both humans and animal studies [4,5].

Cornelia Pirulli and Anna Fertonani contributed equally to this work.

The authors declare no conflict of interest.

* Corresponding author. Neuroscience Section, Department of Clinical and Experimental Sciences, University of Brescia, Viale Europa 11; 25123 Brescia, Italy. Tel.: +39 (0)303501597; fax: +39 (0)303533513.

E-mail address: carlo.miniussi@cognitiveneuroscience.it (C. Miniussi).
PL in adult humans has been documented for many perceptual tasks in the auditory, somatosensory and visual domains, and the neural modifications that occur during PL are direct evidence of the presence of cortical plasticity in the brain [4-7]. In a recent study, we demonstrated that the application of tES to the primary visual cortex (V1) during a visual PL task [8-10] enhances the behavioral performance [11]. In the same stream, in the present study, we aim to determine if there is an ideal timing (before or during task execution) for the application of different types of tES to induce neuromodulation in the adult human cerebral cortex.

Different types of tES are differentiated by specific modalities of current discharge (e.g., direct versus alternating) that might have different neuromodulatory effects at neuronal level. In this work, we applied two types of non-invasive tES, direct current stimulation (tDCS) and random noise stimulation (tRNS). tDCS is a technique that permits the modulation of cortical excitability in 
a polarity-specific manner (anodal versus cathodal) [12]. In tDCS, a continuous direct current of low-level intensity ( 1-2 $\mathrm{mA}$ ) crosses the scalp and stimulates the cerebral cortex, modulating the neural activity in the stimulated area [1]. tDCS effects are most likely induced by membrane polarization, altering the firing rates of neurons. Anodal tDCS induces depolarization, while cathodal tDCS induces hyperpolarization [13]. In tRNS, a repetitive alternating current of different frequencies in a random mode is applied to the cortex through the scalp [11,14]. In contrast to tDCS, the current flow of tRNS has no directionality. It can be applied at different frequency band ranges from 0.1 to $640 \mathrm{~Hz}$ (lf-tRNS, low frequency from 0.1 to $100 \mathrm{~Hz}$, or hf-tRNS, high frequency from 101 to $640 \mathrm{~Hz}$ ). Terney et al. [14] reported that tRNS improved the performance of implicit motor learning tasks and increased motor cortex excitability. Therefore, we can presume that tRNS, like tDCS, can change cortical excitability by inducing depolarization.

In a precedent-setting report [11], we compared cathodal and anodal tDCS (a-tDCS) and lf-tRNS and hf-tRNS and demonstrated that hf-tRNS is superior to all other methods of stimulation for improving behavioral performance in a visual PL task. This effect was obtained when all tES methods were applied during the execution of a task, confirming that both tDCS and tRNS can induce modulation of behavioral performance, but to different extent. Nevertheless differential effect due to different tES types is not the only issue; these effects may also differ depending on the specific parameters of application like the timing. Therefore, it is important to obtain a better understanding of some of the basic methodological aspects of neuro-modulation induction by tES.

Recently, tES was shown to cause different effects in a motor learning task depending on the timing of the application of stimulation [15]. In a previous study, Nitsche et al. [16] demonstrated that the application of a-tDCS during the execution of an implicit learning task led to an improvement in the rate of learning of the task. By contrast, if 10 min of tES was applied before the same task, no effect on the rate of learning was observed [17]. Supporting this result, Stagg et al. [15] analyzed the responses to an explicit motor learning task performed during or after tDCS, revealing that a-tDCS increased the rate of motor learning only when applied during the execution of the task. However, the extent to which these findings are generalizable and transferable to other areas of the cerebral cortex is unclear, because other studies have reported that the application of a-tDCS before task execution induces behavioral facilitation $[18,19]$.

Given the importance of timing to the relationship between task execution and tDCS, we wondered if the application of different types of tES (tRNS in addition to tDCS) at different times with respect to the task execution would modulate subsequent performance? If the application of tRNS before task execution induces a depolarization in a manner similar to that of tDCS, it should increase the excitability of the system or decrease the threshold response. This effect will 'prime' the system for subsequent behavioral facilitation. However, different neural mechanisms are thought to underlie the action of the two tES techniques. Depolarization in hf-tRNS should be characterized by temporal neural summation because the stimulation causes the membrane potential of the stimulated neurons to approach their response thresholds due to the close temporal sequence of the stimuli [14]. This temporal summation would reinforce the activity only of the neurons engaged in the task execution, inducing an enhancement of the behavioral performance [11]. This effect should stop as soon the stimulation stops because the timing of the neural on-going activity is a fundamental aspect of the temporal summation concept. Consequently we expect to observe a performance improvement if hf-tRNS is applied during but not before the visual PL. Conversely, depolarization induced by a-tDCS, in which neurons are exposed to a constant electric field, should rely mainly on the initiation of homeostatic mechanisms. These homeostatic mechanisms might not be totally functional if at play during the task execution, but they should, eventually, induce stronger aftereffects. Therefore, the a-tDCS effect should be carried over after the end of stimulation and we expect to see an improvement in the performance mainly if a-tDCS is applied before the task.

Starting from these considerations, the principal aim of this work was to determine if there was an ideal timing for the application of different types of facilitatory tES to induce neuromodulation in the V1 cortex. These results were expected to provide important guidelines for the design of rehabilitation protocols in clinical neuroscience [20] by determining which of the two excitatory techniques is more effective and the optimal timing of each technique. Hf-tRNS was expected to be effective only if applied during task execution, whereas a-tDCS was expected to induce a stronger facilitation when applied before task execution.

\section{Materials and methods}

\section{Subjects}

A total of 101 healthy subjects participated in the experiment. All of the participants were right-handed with normal or corrected-tonormal vision. Subjects with a history of seizures, implanted metal objects, heart problems or any neurological disease were not included. Moreover, subjects who performed below chance (no learning) were excluded from the study. Based on these criteria, 11 participants were excluded. The remaining 90 subjects (45 males, mean age \pm standard deviation $21.8 \pm 2.9$ years; range $19-32$ years) participated in the experiment. The subjects were assigned to one of six groups: two groups received online stimulation (online-hftRNS; online-a-tDCS), two groups received offline stimulation (offline-hf-tRNS; offline-a-tDCS), and two control groups received placebo stimulation (online-sham; offline-sham). Each group, with the exception of the offline-sham group, included 14 participants ( 7 males and 7 females), and the mean ages of the groups were $22.4 \pm 2.8$ years for the online-hf-tRNS group, $21.8 \pm 2.3$ years for the online-a-tDCS group, $21.6 \pm 2.7$ years for the offline-hf-tRNS group, $21.7 \pm 3.4$ years for the offline-a-tDCS group and $21.6 \pm 3.0$ years for the online-sham group. The offline-sham condition included 10 participants ( 5 males and 5 females), with a mean age of $21.9 \pm 3.6$ years. The data for the three online conditions were collected in a previous experiment (for details see [11]).

The study was approved by the Ethics Committee of the IRCCS Centro San Giovanni di Dio Fatebenefratelli, Brescia, Italy. Safety procedures were adopted based on non-invasive brain stimulation approaches [21,22], and informed consent was obtained from all participants prior to the beginning of the experiment.

\section{Ovarian hormone influence on data variability}

tDCS studies [17,23] have highlighted gender differences in both visual and motor domains. Some of these differences are likely due to the influence of ovarian hormones on task performance, as has been demonstrated by several transcranial magnetic stimulation (TMS) studies [24-27]. To control for the gender differences in this study, the female participants were tested during the follicular menstrual phase (mean day from the first day of the menstrual period: $14.8 \pm 3.0$, range $10-20$ days) because progesterone levels are low and estrogen levels are high during this period. Under these conditions, the cortical excitability of male and female subjects is thought to be similar [26]. 


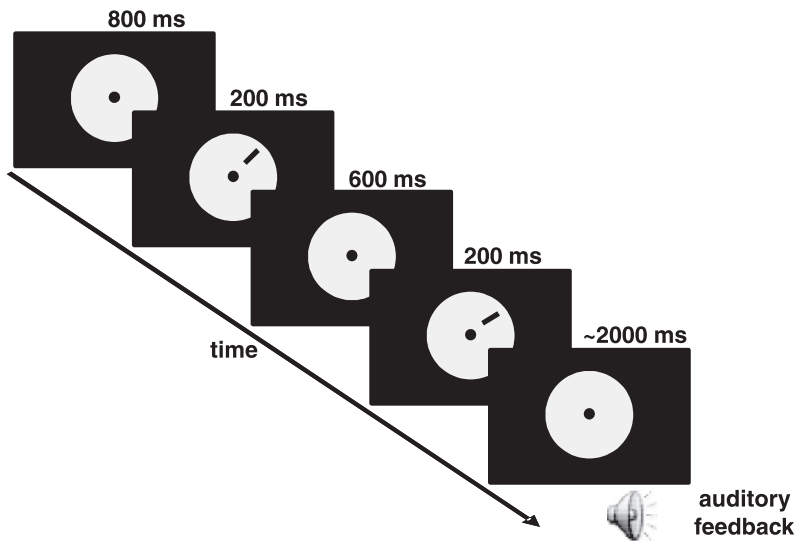

Figure 1. Trial structures. Example of a trial of the orientation discrimination task with the reference and target stimuli presented in the upper-right hemi-field.

\section{Orientation discrimination task (ODT)}

The participants were seated in front of a computer screen in a quiet, semi-dark room. A chin rest was used to maintain a distance of $57 \mathrm{~cm}$ between the participant and the screen. In the ODT, participants had to decide whether the presented stimulus was tilted clockwise or counterclockwise relative to the previously presented stimulus. The subjects were asked to respond as quickly and accurately as possible after the second stimulus was presented by pressing the left (counterclockwise) or right (clockwise) button of a response pad with the left or right index finger, respectively. Auditory feedback (duration $=50 \mathrm{~ms}$; frequency for the correct response $=700 \mathrm{~Hz}$; frequency for the incorrect response $=350 \mathrm{~Hz}$ ) informed the subjects about the correctness of their responses. All stimuli were black lines, and each line stimulus was $2^{\circ}$ long and 5 min wide (in visual angles). The orientation of one of the two lines of the couple was fixed, and was $45^{\circ}$ in the upper right and lower left hemi-fields and $135^{\circ}$ in the upper left and lower right hemifields. One of these lines, with fixed orientation, was presented first in half of the trials and second in the other half of the trials. The angular differences between the fixed orientation line and the other line could be of $\pm 1.10,1.21,1.33$ and $1.46^{\circ}$ [28]. All of the above experimental parameters were balanced and randomized between blocks (see trial structure in Fig. 1).

Stimuli were presented on a computer screen using Presentation software v. 12.0 (http://www.neurobs.com) in each of the four visual hemi-fields: upper left, upper right, lower left and lower right. In each trial, the two stimuli were presented in the same hemi-field (Fig. 1). To limit the area in which the stimuli were presented, a black piece of cardboard covered the screen except for a $10-\mathrm{cm}$-diameter circle located at the center of the screen. A central fixation point was maintained for the duration of the trial.

Each block of the ODT consisted of 64 trials and lasted approximately $4 \mathrm{~min}$. The ODT consisted of five experimental blocks plus a training block. The training block was similar to the experimental blocks but contained a different number of trials (only 8 ) and an increased rotation angle between the two stimuli ( $15^{\circ}$ clockwise or counterclockwise).

\section{Transcranial electric stimulation techniques: tRNS and tDCS}

tDCS and tRNS were delivered by a battery-driven stimulator (Eldith-Plus, NeuroConn GmbH, Ilmenau, Germany) through a pair of saline-soaked sponge electrodes. The active electrode $\left(16 \mathrm{~cm}^{2}\right)$ was placed over the occipital cortex in the area corresponding to V1, which was defined as $10 \%$ of the nasion-inion distance above the inion (mean position $=3.5 \pm 0.2 \mathrm{~cm}$ above the inion). The reference electrode $\left(60 \mathrm{~cm}^{2}\right)$ was fixed extracephalically on the right arm. The electrodes were kept in place with elastic bands, and an electroconductive gel was applied under the electrodes before application to reduce skin impedance. The intensity of stimulation was $1.5 \mathrm{~mA}$, and the current density varied between 25 and $94 \mu \mathrm{A} / \mathrm{cm}^{2}$. When tDCS was applied, the polarity of the active electrode was anodal, whereas the two electrodes were not polarity dependent for tRNS. tRNS consisted of an alternating current with a $0 \mathrm{~mA}$ offset applied at random frequencies (range 101-640 Hz, i.e., hf-tRNS). The stimulation did not induce any phosphene perception [29]. In the sham stimulations, the current was turned off $20 \mathrm{~s}$ after the stimulation began [30].

In the online conditions, the stimulations were applied for approximately 4 min during each of the five experimental blocks. The total duration of the stimulations was approximately $22 \mathrm{~min}$. In the offline conditions, the stimulations were applied before the execution of the task while the subjects listened to an audio book played on an audio device, maintaining the same time intervals used in the online condition (i.e., $\sim 4$ min of stimulations -2 min of pause $-\sim 4$ min of stimulation and so on). The duration of the entire experimental session was approximately $30 \mathrm{~min}$ for the online conditions and approximately $60 \mathrm{~min}$ for the offline conditions. The procedure is described in Fig. 2.

Although the sham condition should be identical regardless of the timing of application, we have chosen to apply it both in online and offline conditions as an additional control.

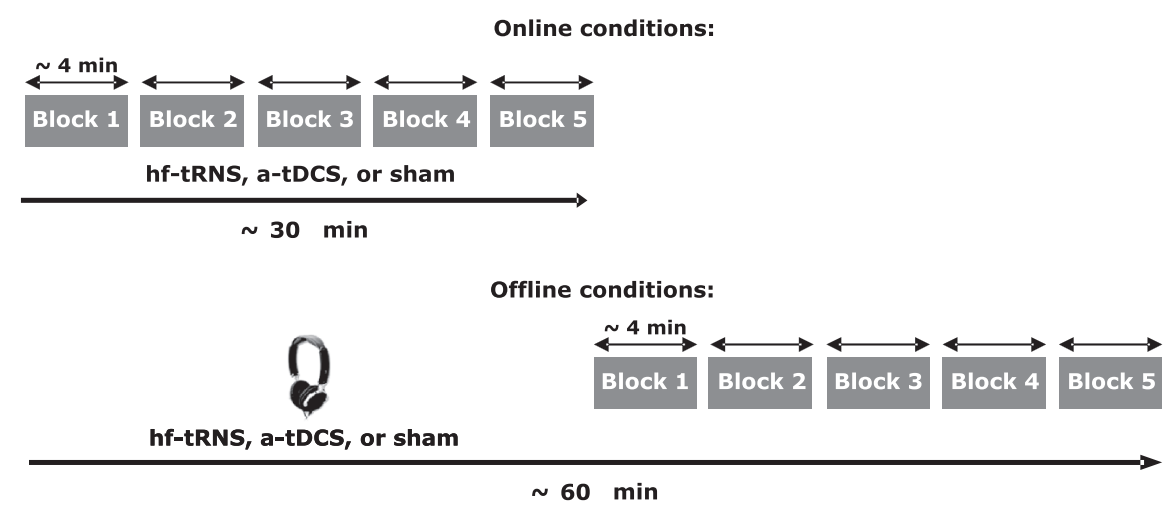

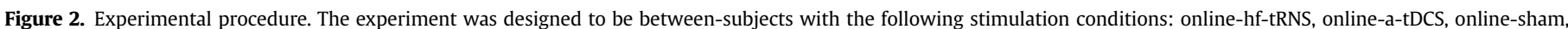

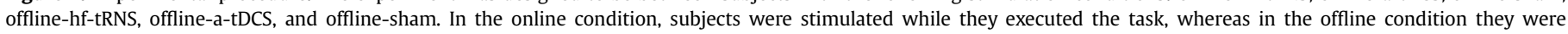

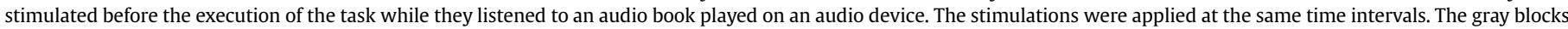
represent the blocks of the orientation discrimination task. 


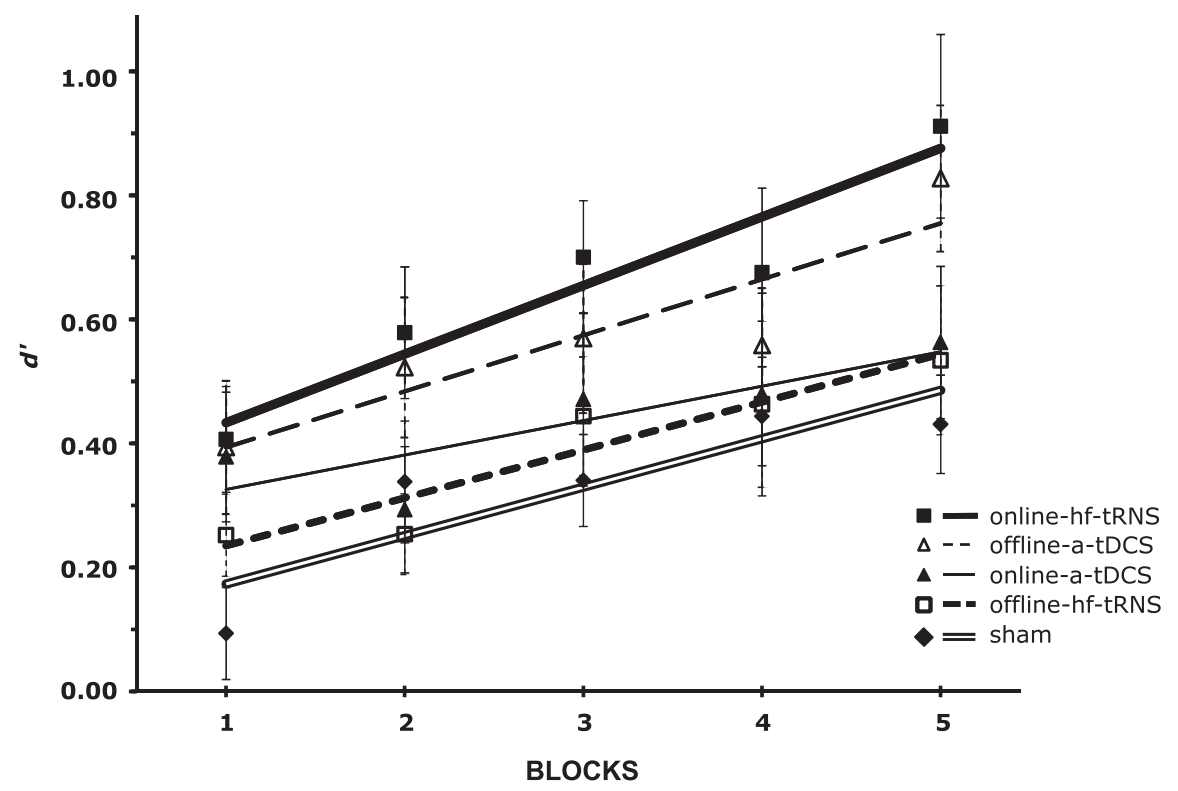

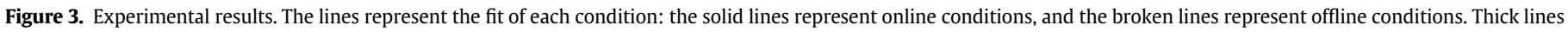
correspond to hf-tRNS, while thin lines represent a-tDCS. The double line represents the sham condition (mean of online- and offline-sham).

At the end of the experimental session, we asked all subjects to complete a questionnaire developed by our research laboratory about the sensations they experienced during the different stimulations [19].

In conclusion, the experiment was a between-subjects design with six stimulation conditions: online-hf-tRNS, online-a-tDCS, online-sham stimulation, offline-hf-tRNS, offline-a-tDCS, and offline-sham stimulation.

\section{Data analysis}

The average orientation sensitivity was calculated as a d' value for each subject and each block separately for each stimulation condition. Moreover we have compared the overall performance improvement in each condition of stimulation ( $d$ '-block $5-d$ 'block 1).

The Kolmogorov-Smirnov test confirmed the normality of the distribution of d' values, and the data were analyzed using a repeated-measures analysis of variance (ANOVA). The data sphericity was tested using the Mauchly test, where appropriate. When the test results were statistically significant, the data were corrected using the Huynh-Feldt correction. The effect size is reported using the partial Eta squared value. A $P$-value $<0.05$ was considered significant for all statistical analyses. For multiple comparisons, we used Fisher's Least Significant Difference (LSD) method to test our specific "a priori" hypotheses (i.e., to compare the different stimulation conditions and different timings of application). For all other comparisons, the $P$-values were corrected using a Bonferroni correction.

Data from the sensations induced by tES were analyzed using the Kruskal-Wallis one-way analysis of variance and, consequently, with multiple comparisons.

\section{Results}

Orientation sensitivity - d'

We first verified that there was no difference between the two sham conditions. A repeated-measures ANOVA with block (from 1 to 5 ) as a within-subjects factor and stimulation condition (online- sham, offline-sham) as a between-subjects factors confirmed the absence of statistically significant differences between the two sham conditions $[F(1,22)=0.29 ; P=0.59]$. For this reason, we collapsed the two conditions into a single sham condition.

We performed a repeated-measure ANOVA with block (from 1 to 5) as a within-subjects factor and stimulation condition (online-hftRNS, online-a-tDCS, offline-hf-tRNS, offline-a-tDCS, and sham) and gender (male, female) as between-subjects factors. We observed a significant main effect for block $[F(4,280)=17.95 ; P<0.01$; $\left.\eta_{\mathrm{P}}^{2}=0.20\right]$, stimulation condition $[F(4,70)=3.02 ; P=0.02$; $\left.\eta_{\mathrm{P}}^{2}=0.15\right]$ and gender $\left[F(1,70)=5.31 ; P=0.02 ; \eta_{\mathrm{P}}^{2}=0.07\right]$. The interaction between block and stimulation condition was not statistically significant $[F(16,280)=1.03 ; P=0.43]$.

For block, multiple post-hoc comparisons revealed a statistically significant difference between block 1 and blocks $3(P<0.01)$, $4(P<0.01), 5(P<0.01)$, between block 2 and blocks $4(P=0.04)$, $5(P<0.01)$, and between blocks 3 and $5(P=0.01)$.

For stimulation conditions, multiple post-hoc comparisons revealed that online-hf-tRNS (mean $d^{\prime} \pm$ standard error of the mean - SEM $=0.625 \pm 0.121$ ) was significantly different from offline-hf-tRNS $(0.389 \pm 0.102)$ and sham $(0.329 \pm 0.067)$ (respectively $P=0.03$ and $P<0.01$ ) and marginally different from online a-tDCS $(P=0.07)(0.437 \pm 0.127)$. Moreover, offline-a-tDCS $(0.574 \pm 0.112)$ was different from sham $(P=0.02)$ (see Fig. 3 ).

The gender factor was statistically significant. Males were more accurate than females in all conditions (males: $0.546 \pm 0.065$; females: $0.371 \pm 0.073$ ). The interaction between the gender and stimulation condition factors was statistically significant $\left[F(4,70)=2.90 ; P=0.03 ; \eta_{\mathrm{P}}^{2}=0.14\right]$. However, post-hoc comparisons did not demonstrate any relevant results.

In addition we wanted to verify the presence of learning in each single stimulation condition. First we have tested if there were significant differences in overall performance improvements for the different stimulation conditions. We performed a one-way ANOVA on the absolute change in $d^{\prime}$ over time ( $d$ '-block 5 - $d$ '-block 1 ). The stimulation condition factor was not statistically significant $[F(4,75)=1.64 ; P=0.17]$.

To evaluate the effect of stimulation at different time points of the protocol (blocks 1 and 5: beginning and end of stimulation, 
Table 1

d' data for all experimental conditions.

\begin{tabular}{|c|c|c|c|c|c|}
\hline Stimulation condition & Block 1 mean $( \pm$ SEM $)$ & Block 2 mean $( \pm$ SEM $)$ & Block 3 mean $( \pm$ SEM $)$ & Block 4 mean $( \pm$ SEM $)$ & Block 5 mean $( \pm$ SEM $)$ \\
\hline Sham & $0.09( \pm 0.07)$ & $0.34( \pm 0.10)$ & $0.34( \pm 0.07)$ & $0.44( \pm 0.08)$ & $0.43( \pm 0.08)$ \\
\hline \multicolumn{6}{|l|}{ Online } \\
\hline a-tDCS & $0.38( \pm 0.10)$ & $0.29( \pm 0.10)$ & $0.47( \pm 0.14)$ & $0.48( \pm 0.16)$ & $0.56( \pm 0.12)$ \\
\hline hf-tRNS & $0.41( \pm 0.09)$ & $0.58( \pm 0.11)$ & $0.70( \pm 0.09)$ & $0.68( \pm 0.14)$ & $0.91( \pm 0.15)$ \\
\hline \multicolumn{6}{|l|}{ Offline } \\
\hline a-tDCS & $0.39( \pm 0.11)$ & $0.52( \pm 0.11)$ & $0.57( \pm 0.12)$ & $0.56( \pm 0.09)$ & $0.83( \pm 0.12)$ \\
\hline hf-tRNS & $0.25( \pm 0.07)$ & $0.25( \pm 0.07)$ & $0.44( \pm 0.10)$ & $0.46( \pm 0.13)$ & $0.53( \pm 0.12)$ \\
\hline
\end{tabular}

respectively), we compared the different stimulations by separately considering each block. In block 1 , there was a significant main effect of the stimulation condition $[F(4,75)=2.80 ; P=0.03$; $\left.\eta_{\mathrm{p}}^{2}=0.13\right]$. Post-hoc comparisons revealed differences between sham and online-a-tDCS, offline-a-tDCS and online-hf-tRNS (respectively $P=0.02, P=0.01$ and $P=0.01$ ). In block 5, there was a significant main effect of the stimulation condition $\left[F(4,75)=3.41 ; P=0.01 ; \eta_{\mathrm{p}}^{2}=0.15\right]$. Post-hoc comparisons revealed a difference between the sham condition and online-hftRNS and offline-a-tDCS (respectively $P<0.01$ and $P=0.01$ ). Moreover, online-hf-tRNS was different from offline-hf-tRNS and online-a-tDCS (respectively $P=0.03$ and $P=0.04$ ) (see Table 1 ).

Successively, we performed a repeated measures ANOVAs separately for each stimulation condition. The $P$-values were corrected for multiple comparisons ( $P$ considered significant if $<0.01$ [0.05/5]). These analyses shown the main effect of block for all stimulation conditions, with the exception of online-a-tDCS and offline-hf-tRNS. Post-hoc comparisons in the online-hf-tRNS condition $\left[F(4,52)=7.62 ; P<0.01 ; \eta_{\mathrm{p}}^{2}=0.37\right]$ revealed that block 1 was different from blocks $3(P<0.01), 4(P=0.07)$ and $5(P<0.01)$, and blocks 2,3 and 4 differed from block $5(P<0.01$, $P=0.03, P=0.02$ respectively). In the offline-a-tDCS condition $\left[F(4,52)=6.78 ; P<0.01 ; \eta_{\mathrm{P}}^{2}=0.34\right]$, block 1 was different from blocks $3(P=0.05), 4(P=0.06)$ and $5(P<0.01)$, and blocks 2,3 and 4 differed from block 5 ( $P<0.01$ for each comparison). In the sham condition $\left[F(4,92)=6.33 ; P<0.01 ; \eta_{\mathrm{P}}^{2}=0.22\right]$, block 1 was different from blocks $2,3,4$ and 5 ( $P<0.01$ for each comparison). No statistically significant differences were observed in the onlinea-tDCS condition $[F(4,52)=1.29 ; P=0.29]$ and in the offline-hftRNS condition $[F(4,52)=3.42 ; P=0.014]$, which suggested the absence of an enhancement statistically significant in these two stimulation conditions.

The present data support the initial hypothesis that stimulation timing is important. The online-hf-tRNS was more effective than offline-hf-tRNS. By contrast, offline-a-tDCS was more effective than online-a-tDCS.
Sensations induced by different types of tES

Each participant completed a questionnaire at the end of the experiment [19] and reported having tolerated the stimulation without discomfort. The results of the questionnaire are reported in Table 2. The participants were unable to distinguish the real stimulation from the placebo stimulation. The non-parametric Kruskal-Wallis test was performed to compare the different stimulations for each sensation. The analysis highlighted no difference between the stimulations for pain, heat, iron taste, and fatigue sensations. Interestingly, the analysis demonstrated a statistically significant difference between the stimulations with respect to irritation $[H(4, N=80)=35.15, P<0.01]$, burning $[H(4, N=80)=28.47, P<0.01]$ and itching $[H(4, N=80)=35.30$, $P<0.01]$. Subsequently, multiple comparisons were performed for these three sensations. For irritation, itching and burning, online-atDCS and offline-a-tDCS were significantly different (all P's $<0.05$ ) from sham, online-hf-tRNS and offline-hf-tRNS.

In general, the tDCS-induced sensations were perceived more strongly [21,31] than the tRNS- or sham-induced sensations; moreover offline-a-tDCS was perceived most strongly than onlinea-tDCS. By contrast, hf-tRNS was indistinguishable from sham conditions for all of the sensations examined. This characteristic makes tRNS an optimal tool for experiments in which sham stimulations must not be distinguishable from real stimulations [31].

\section{Discussion}

The main result of this study is the finding that the timing of identical tES protocols yields opposite effects on performance. Therefore, the efficacy with which different types of tES induce neural modulation differ according to the excitability levels of the stimulated neurons at the time of stimulation application. These differences are reflected by different levels of behavioral performance under different experimental conditions.

Table 2

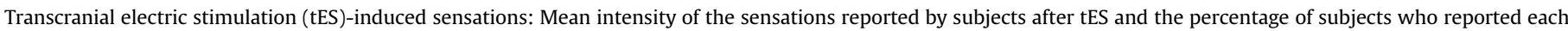

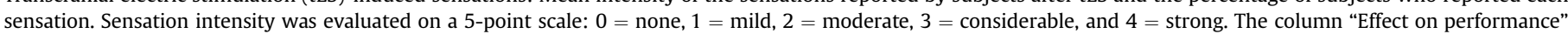
indicates the subjective feelings of the participants relative to the effect of the tES-induced sensation on performance.

\begin{tabular}{|c|c|c|c|c|c|c|c|c|}
\hline Stimulation condition & Irritation & Pain & Burning & Heat & Itch & Iron taste & Fatigue & Effect on performance \\
\hline \multicolumn{9}{|l|}{ Sham } \\
\hline Intensity & 0.3 & 0.0 & 0.2 & 0.2 & 0.5 & 0.1 & 0.3 & - \\
\hline Subjects (\%) & 25 & 0 & 17 & 17 & 38 & 4 & 25 & - \\
\hline \multicolumn{9}{|l|}{ Online a-tDCS } \\
\hline Intensity & 1.1 & 0.2 & 0.7 & 0.4 & 1.4 & 0.1 & 0.4 & 0.5 \\
\hline Subjects (\%) & 79 & 14 & 50 & 29 & 86 & 7 & 29 & 36 \\
\hline \multicolumn{9}{|l|}{ Online hf-tRNS } \\
\hline Intensity & 0.1 & 0.0 & 0.1 & 0.3 & 0.3 & 0.0 & 0.5 & 0.4 \\
\hline Subjects (\%) & 14 & 0 & 7 & 21 & 29 & 0 & 43 & 36 \\
\hline \multicolumn{9}{|l|}{ Offline a-tDCS } \\
\hline Intensity & 1.8 & 1.0 & 1.5 & 1.3 & 1.9 & 1.5 & 1.0 & - \\
\hline Subjects (\%) & 86 & 14 & 79 & 29 & 100 & 14 & 14 & - \\
\hline \multicolumn{9}{|l|}{ Offline hf-tRNS } \\
\hline Intensity & 1.0 & 0.5 & 0.5 & 0.8 & 0.7 & 0.1 & 1.7 & - \\
\hline Subjects (\%) & 14 & 14 & 14 & 36 & 21 & 7 & 21 & - \\
\hline
\end{tabular}


We observed a significant improvement in performance when atDCS was applied before the task (i.e., offline) but not when it was applied during the task execution (i.e., online). On the contrary, performance improved when hf-tRNS, was applied during the task (i.e., online) but not when it was applied before the task (i.e., offline). We conclude that the effects of tES are highly dependent on the timing of the stimulation with respect to the neural activation state. These results confirm our hypothesis that the state of cerebral activation when the non-invasive brain stimulation is applied is important for inducing differential effects on behavioral performance.

The relationship between timing and motor learning has been examined previously [15-17], with effects that differed from those revealed in this work. Nitsche and colleagues [16] initially demonstrated a facilitation effect of anodal stimulation applied during an implicit motor learning task. Subsequently Kuo et al. [17] investigated the effect of tDCS on the same task when applying an offline stimulation before task execution, revealing that a-tDCS had an effect only when it was applied together with a partial NMDAreceptor agonist. When tDCS was applied without drugs, no effect of anodal or cathodal stimulation was observed. Recently, Stagg et al. [15] investigated the timing-dependence of interactions between a-tDCS and learning in an explicit motor learning paradigm. In that study, $10 \mathrm{~min}$ of a-tDCS $(1 \mathrm{~mA})$ increased the rate of learning if applied during the execution of the task but not if applied before the task. These two studies suggest that a-tDCS should be applied online to produce facilitation in a motor task. However, as we have shown, these data are not generalizable to the visual cortex. The disagreement between motor and visual studies may be explained by the differences between the two neural systems. Indeed, results obtained for the motor cortex do not always agree with results obtained for the visual cortex [32] or other areas [33] because there are cyto- and myeloarchitectonic differences between these areas, including differences in neuronal diameters, that may result in a differential current diffusion [34-36]. Besides, differential effects can also be explained by indirect effects due to modulation of other areas via the primary motor cortex (e.g., basal ganglia, premotor or supplementary motor areas).

In the present study, we have demonstrated that the effect of a specific tES differs depending on the timing of application. Our data reveal that the effect of a-tDCS is greater if applied offline than online. We did observe an initial facilitation with online-a-tDCS. However, this initial effect was followed by an absence of improvement in the subsequent blocks, an effect that may be due to the activation of homeostatic mechanisms. The initial improvement of performance could be caused by the strengthening of the neural circuitry provoked by the neuronal membrane depolarization induced by a-tDCS. Both the prolonged delivery of a-tDCS and the sustained exposure to a stimulus cause a sustained depolarization, with an intracellular increase of $\mathrm{Na}^{+}$and $\mathrm{K}^{+}$. Excessive intracellular concentrations of these ions can induce saturation in the neuron and the activation of self-regulatory mechanisms involving voltagedependent channels [37]. The resulting rebalancing of voltagedependent ion channel conductance (in particular $\mathrm{Na}^{+}$and $\mathrm{K}^{+}$ channels) prevents further improvement in behavioral performance. Therefore an initial facilitatory effect might reverse in inhibitory in case of longer stimulation periods. These saturation mechanisms may not occur when the stimulation is given alone namely before the task. Therefore, the facilitation effect of offline-atDCS may be caused by an optimal level of intracellular $\mathrm{Ca}^{2+}$ and $\mathrm{Na}^{+}$.

Although the delivery of offline-a-tDCS induced a facilitation effect, this effect did not reach the same level of facilitation that was obtained with online-hf-tRNS. The more robust effect of online-hf-RNS could be explained by the hypothesis of temporal summation and interaction with task-induced activity. The repetitive and random wave shape of tRNS would induce temporal summation of neural activity if the time-constant of a neuron is sufficiently long to permit the summation of two stimuli presented in close sequence. Terney et al. [14] suggested that $\mathrm{Na}^{+}$-channel activity can be augmented during tRNS. After a depolarization, depolarization of $\mathrm{Na}^{+}$channels would generally take some time, but if a repeated stimulation is applied, these channels can be reopened in a shorter time [38]. The interval at which the pulses are repeated must be short and related to the time constant of the nerve membrane [14]. We speculate that hf-tRNS, with a frequency range between 100 and $640 \mathrm{~Hz}$, may be optimal to interact with neural activity because this type of stimulation approaches the time constant of the cell body and dendrites, between 1 and $10 \mathrm{~ms}$ [39]. Based on the same logic, hf-tRNS should increase the stability of learning. If the stimulated neural population is not involved in the task execution, and consequently there is no task-related neural activity, the effects of hf-tRNS should be null [11]. This is confirmed by the absence of an effect with offline-hf-tRNS.

In addition, it is important to consider that the effects of tES depend on several factors, both internal and external to the technique. Technical internal factors include the current density, the location of the reference electrode, the duration of the stimulation, the intervals between stimulations and the timing of the stimulation. External factors include the cortical area stimulated, the task used and the subject characteristics (e.g., healthy young versus elderly subjects).

The pattern of stimulation delivery, such as the length of pauses between blocks, is also an important factor. In the present experiment, the stimulation was applied in blocks of 4 min separated by 2-min intervals. In light of recently published studies [40-42], the presence of the pauses may be a key variable that determines the final induced effect. The impact of tDCS on the neural activity may differ depending on the length of the breaks between stimulations and the duration of the stimulation. Fricke et al. [40] explored the dependence of homeostatic plasticity on the time interval between the application of two protocols. The authors measured the motor evoked potential amplitude after repeated tDCS stimulation of the motor cortex and compared the effect of a single 5 min session of anodal or cathodal tDCS with the effect of a 5 min session preceded by an identical 5 min conditioning session 30,3 or 0 min beforehand. A repeated short period of motor cortex tDCS follows a timedependent rule that is compatible with homeostatic mechanisms. Five minutes of anodal tDCS increased neuronal excitability for approximately $5 \mathrm{~min}$. However, if two 5 min periods of tDCS are applied with a 3 min interval, the second session has the opposite effect. Moreover, Monte-Silva et al. [42] showed that the application of a second period of stimulation during the after-effects of a first period ( $13 \mathrm{~min}$ of stimulation with breaks of $3 \mathrm{~min}, 20 \mathrm{~min}, 3 \mathrm{~h}$, or $24 \mathrm{~h}$ ) initially causes a reduction in the amplitude of motor evoked potentials but then increases excitability. These data highlight the importance of inter-stimulation intervals. These results are not surprising, considering the abundant evidence in the literature of the phenomena of meta-plasticity when applying non-invasive brain stimulation (i.e., TMS, tDCS) [43-46].

The major objective of our work was to establish the ideal timing of stimulation for different types of tES. We demonstrated that the application of hf-tRNS during task execution maximizes the capacity of neural circuits to change their activity over time to increase the efficiency of behavioral performance. Conversely, the beneficial effects of a-tDCS were maximized when applied before task execution. In conclusion, our work highlights the specificity of the effects induced by hf-tRNS and a-tDCS as a function of the state of activation of the engaged neuronal population. These data have important implications for basic neuroscience as well as neurorehabilitation because they may enable the targeting of specific 
cognitive and behavioral functions for specific enhancement with ideal protocols as a component of therapeutic intervention.

\section{Acknowledgments} Health.

This work was supported by a grant from the Italian Ministry of

\section{References}

[1] Nitsche MA, Paulus W. Transcranial direct current stimulation - update 2011. Restor Neurol Neurosci 2011;29(6):463-92.

[2] Paulus W. Transcranial electrical stimulation (tES - tDCS; tRNS, tACS) methods. Neuropsychol Rehabil 2011;21(5):602-17.

[3] Miniussi C, Vallar G. Brain stimulation and behavioural cognitive rehabilitation: a new tool for neurorehabilitation? Neuropsychol Rehabil 2011;21(5): 553-9.

[4] Fahle M, Poggio T, editors. Perceptual learning. Cambridge, Massachusetts: MIT Press; 2002.

[5] Gilbert CD, Sigman M, Crist RE. The neural basis of perceptual learning. Neuron 2001;31(5):681-97.

[6] Carmel D, Carrasco M. Perceptual learning and dynamic changes in primary visual cortex. Neuron 2008;57(6):799-801.

[7] Li W, Piech V, Gilbert CD. Perceptual learning and top-down influences in primary visual cortex. Nat Neurosci 2004;7(6):651-7.

[8] Furmanski CS, Schluppeck D, Engel SA. Learning strengthens the response of primary visual cortex to simple patterns. Curr Biol 2004;14(7):573-8.

[9] Seitz A, Watanabe T. A unified model for perceptual learning. Trends Cogn Sci 2005;9(7):329-34.

[10] Thiele A. Perceptual learning: is V1 up to the task? Curr Biol 2004;14(16): R671-3.

[11] Fertonani A, Pirulli C, Miniussi C. Random noise stimulation improves neuroplasticity in perceptual learning. J Neurosci 2011;31(43):15416-23.

[12] Nitsche MA, Paulus W. Excitability changes induced in the human motor cortex by weak transcranial direct current stimulation. J Physiol 2000; 527(Pt 3):633-9.

[13] Liebetanz D, Nitsche MA, Tergau F, Paulus W. Pharmacological approach to the mechanisms of transcranial DC-stimulation-induced after-effects of human motor cortex excitability. Brain 2002;125(Pt 10):2238-47.

[14] Terney D, Chaieb L, Moliadze V, Antal A, Paulus W. Increasing human brain excitability by transcranial high-frequency random noise stimulation. J Neurosci 2008;28(52):14147-55.

[15] Stagg CJ, Nitsche MA. Physiological basis of transcranial direct current stimulation. Neuroscientist 2011;17(1):37-53.

[16] Nitsche MA, Schauenburg A, Lang N, Liebetanz D, Exner C, Paulus W, et al. Facilitation of implicit motor learning by weak transcranial direct current stimulation of the primary motor cortex in the human. J Cogn Neurosci 2003; 15(4):619-26.

[17] Kuo MF, Paulus W, Nitsche MA. Sex differences in cortical neuroplasticity in humans. Neuroreport 2006;17(16):1703-7.

[18] Vallar G, Bolognini N. Behavioural facilitation following brain stimulation: implications for neurorehabilitation. Neuropsychol Rehabil 2011;21(5): 618-49.

[19] Fertonani A, Rosini S, Cotelli M, Rossini PM, Miniussi C. Naming facilitation induced by transcranial direct current stimulation. Behav Brain Res 2010; 208(2):311-8.

[20] Miniussi C, Cappa SF, Cohen LG, Floel A, Fregni F, Nitsche M, et al. Efficacy of repetitive transcranial magnetic stimulation/transcranial direct current stimulation in cognitive neurorehabilitation. Brain Stimul 2008;1(4):326-36.

[21] Poreisz C, Boros K, Antal A, Paulus W. Safety aspects of transcranial direct current stimulation concerning healthy subjects and patients. Brain Res Bull 2007;72(4-6):208-14.

[22] Rossi S, Hallett M, Rossini PM, Pascual-Leone A, Safety of TMS Consensus Group. Safety, ethical considerations, and application guidelines for the use of transcranial magnetic stimulation in clinical practice and research. Clin Neurophysiol 2009;120(12):2008-39.
[23] Chaieb L, Antal A, Paulus W. Gender-specific modulation of short-term neuroplasticity in the visual cortex induced by transcranial direct current stimulation. Vis Neurosci 2008;25(1):77-81.

[24] Smith MJ, Adams LF, Schmidt PJ, Rubinow DR, Wassermann EM. Effects of ovarian hormones on human cortical excitability. Ann Neurol 2002;51(5): 599-603.

[25] Smith MJ, Keel JC, Greenberg BD, Adams LF, Schmidt PJ, Rubinow DA, et al. Menstrual cycle effects on cortical excitability. Neurology 1999;53(9): 2069-72.

[26] Inghilleri M, Conte A, Curra A, Frasca V, Lorenzano C, Berardelli A. Ovarian hormones and cortical excitability. An rTMS study in humans. Clin Neurophysiol 2004;115(5):1063-8.

[27] Sale MV, Ridding MC, Nordstrom MA. Factors influencing the magnitude and reproducibility of corticomotor excitability changes induced by paired associative stimulation. Exp Brain Res 2007;181(4):615-26.

[28] Matthews N, Liu Z, Geesaman BJ, Qian N. Perceptual learning on orientation and direction discrimination. Vis Res 1999;39(22):3692-701.

[29] Schwiedrzik CM. Retina or visual cortex? The site of phosphene induction by transcranial alternating current stimulation. Front Integr Neurosci 2009;3:6.

[30] Gandiga PC, Hummel FC, Cohen LG. Transcranial DC stimulation (tDCS): a tool for double-blind sham-controlled clinical studies in brain stimulation. Clin Neurophysiol 2006;117(4):845-50.

[31] Ambrus GG, Paulus W, Antal A. Cutaneous perception thresholds of electrical stimulation methods: comparison of tDCS and tRNS. Clin Neurophysiol 2010; 121(11):1908-14.

[32] Antal A, Nitsche MA, Paulus W. Transcranial direct current stimulation and the visual cortex. Brain Res Bull 2006;68(6):459-63.

[33] Jacobson L, Koslowsky M, Lavidor M. tDCS polarity effects in motor and cognitive domains: a meta-analytical review. Exp Brain Res 2011;216(1): $1-10$.

[34] Stuart G, Spruston N. Determinants of voltage attenuation in neocortical pyramidal neuron dendrites. J Neurosci 1998;18(10):3501-10.

[35] Spruston N. Pyramidal neurons: dendritic structure and synaptic integration. Nat Rev Neurosci 2008;9(3):206-21.

[36] Brodal A. Neurological anatomy in relation to clinical medicine. 3rd ed. New York: Oxford University Press; 1981.

[37] Levitan IB, Kaczmarek LK. The neuron. In: Cell and molecular biology. 3rd ed. New York: Oxford University Press; 2002.

[38] Schoen I, Fromherz P. Extracellular stimulation of mammalian neurons through repetitive activation of $\mathrm{Na}+$ channels by weak capacitive currents on a silicon chip. J Neurophysiol 2008;100(1):346-57.

[39] Kandel ER, Schwartz JH, Jessell MT. Principle of neural sciences. 4th ed. McGraw-Hill; 2000.

[40] Fricke K, Seeber AA, Thirugnanasambandam N, Paulus W, Nitsche MA Rothwell JC. Time course of the induction of homeostatic plasticity generated by repeated transcranial direct current stimulation of the human motor cortex. J Neurophysiol 2011;105(3):1141-9.

[41] Monte-Silva K, Kuo MF, Liebetanz D, Paulus W, Nitsche MA. Shaping the optimal repetition interval for cathodal transcranial direct current stimulation (tDCS). J Neurophysiol 2010;103(4):1735-40.

[42] Monte-Silva K, Kuo MF, Hessenthaler S, Fresnoza S, Liebetanz D, Paulus W, et al. Induction of late LTP-like plasticity in the human motor cortex by repeated non-invasive brain stimulation. Brain Stimul 2013;6(3):424-32.

[43] Iyer MB, Mattu U, Grafman J, Lomarev M, Sato S, Wassermann EM. Safety and cognitive effect of frontal DC brain polarization in healthy individuals. Neurology 2005;64(5):872-5.

[44] Siebner HR, Lang N, Rizzo V, Nitsche MA, Paulus W, Lemon RN, et al. Preconditioning of low-frequency repetitive transcranial magnetic stimulation with transcranial direct current stimulation: evidence for homeostatic plasticity in the human motor cortex. J Neurosci 2004;24(13):3379-85.

[45] Gentner R, Wankerl K, Reinsberger C, Zeller D, Classen J. Depression of human corticospinal excitability induced by magnetic theta-burst stimulation: evidence of rapid polarity-reversing metaplasticity. Cereb Cortex 2008;18(9): 2046-53.

[46] Gamboa OL, Antal A, Moliadze V, Paulus W. Simply longer is not better: reversal of theta burst after-effect with prolonged stimulation. Exp Brain Res 2010;204(2):181-7. 